pression is normal in $h f l^{-}$strains, it is possible to exclude the idea that this gene specifies a component of the catabolite repression system. The roles of the catabolite activator protein and the $h f l$ gene product in establishing lysogeny are not clear, but it is likely that these systems may be more intricate than previously suspected, and further components may remain to be identified.

A different form of positive control, so far demonstrated only with the mRNAs of phages T3 and T7, is exercised by processing systems. The RNA polymerase enzyme of the host, $E$. coli, transcribes one large messenger in vitro from the early region of these phages (corresponding to $20 \%$ of the DNA, located at its left end). But infected cells contain five, much smaller, early T7 messengers. Recently it has seemed likely that the discrepancy is due to the presence in the cell of an enzyme, RNase III, which cleaves the large precursor RNA (that is the product in vitro) into mature messengers. Hercules et al. (ibid., 840) now report that this cleavage represents a step essential for phage gene expression, since only the mature mRNAs and not their large precursor, are active as templates for translation in vitro. This concept is supported by the observation that the early phage genes are only poorly translated in RNase III $^{-}$mutants of E. coli. Why the precursor RNA cannot be translated is obscure; but this system may well prove interesting from the viewpoint of translational control as well as for its processing mechanisms. And although no bacterial systems have yet been shown to suffer precursor maturation as a prerequisite for translation, it is an interesting possibility that RNase III may play this part in uninfected bacteria.

Turning to the transcription of $\mathrm{T} 3$ and T7 DNAs which takes place by the phage specified polymerase later during infection, Golomb and Chamberlin (ibid., 769) have mapped the principal units of gene expression. With a template of T7 DNA, the T7 RNA polymerase forms six size classes of mRNA (molecular weights 5.5, 4.5, 2.0, $0.8, \quad 0.4$ and $0.2 \times 10^{6}$ ) equimolar in proportion except for the $2 \times 10^{6}$ species which seems to comprise two classes of mRNA. When the T7 DNA template is shortened by the removal of regions from its right end with nucleases, the smallest mRNA is no longer produced, and the mRNAs of 5.5, 4.5 and (one of) $2.0 \times 10^{6}$ daltons are shortened. Since all these messengers are complementary only to the r-strand of T7 DNA and can be synthesised in spite of deletions at the left end, it seems likely that they are derived from overlapping units of transcription; transcription may start at promotor sites located at 56, 64 and
$83 \%$ along the phage DNA from its left end, and may end at a common termination site near the right end of the molecule.

When T3 DNA is used as a template for the T7 RNA polymerase, a single major RNA species is formed of $2.0 \times 10^{6}$ daltons. Hybrids between T3 and T7 DNA show various patterns of transcription, consistent with an overlapping arrangement of transcription units. Since hybrid templates can be examined by heteroduplex mapping to define precisely which regions are derived from $\mathrm{T} 3$ and which from $\mathrm{T} 7$, future use of this technique may allow the start and stop points of the polymerase to be mapped more precisely. There are two classes of late gene in T7, and so it is tempting to speculate that they may be represented by the two classes of mRNA, one comprising the overlapping transcription units and the other corresponding to the remaining messengers. Although previous suggestions have quite often been made that transcription might start at one point and end at any one of several sites to allow coordinate or noncoordinate expression of adjacent genes, the late phage overlapping messengers seem to achieve the same end by reversing the roles of the start and stop points. It remains to be seen whether this has wider significance for the uninfected bacterium.

\section{How algae survive desiccation}

\section{from Peter D. Moore}

Plant Ecology Correspondent

FEw habitats are subjected to the extremes of environmental conditions experienced by the intertidal zones of coasts. Plants and animals living in these regions face wide fluctuation in temperature, insolation, salinity and desiccation with each ebb and flow of the tide. Most of these organisms are essentially marine and therefore it is the period of emersion which represents the stress phase which must be tolerated to ensure survival. The effect of this stress is not laid evenly over the eulittoral zone, but is most acute at its higher levels, where the emersion period is most protracted. It is this variation of stress intensity with vertical height which, in conjunction with the factor of interspecific competition, accounts for the well known intertidal zonation of benthic marine algae (see Zaneveld, Am. Zool., 9, 367; 1969).

Many of the algae of the upper eulittoral suffer emersion periods of several days, sometimes even weeks, during which they become thoroughly desiccated. The physiological mechanisms which enable these morphologically simple plants to survive such stresses have been a source of interest to botanists for some while.

One aspect of algal physiology which has received attention is that of carbon balance, especially during emersion periods. Chapman (Proc. 5th Int. Seaweed Symp. Halifax N.S., 217; 1966) found that the respiration rate of $\mathrm{Car}$ pophyllum decreases as desiccation proceeds, falling to $20 \%$ of the hydrated rate at $80 \%$ desiccation. Bidwell and Craigie (Can. J. Bot., 41, 179; 1963) also demonstrated that carbon fixation is reduced during emersion in Fucus vesiculosus. Ogata ( $J$. Shimonosaki Univ. Fish., 16, 89; 1968) has since reported that the depression of respiration rate during desiccation is found in many algae, but that rehydration results in an almost instantaneous resumption of normal respiration.

The current Carnegie Institution of Washington Year Book, which covers the years 1972-73, contains information concerning the effect of desiccation on the photochemical reaction of photosynthesis in an intertidal alga. Fork and Hiyama (page 384) have made a study of Porphyra perforata and $P$. tenera, species of the upper eulittoral zone. Under normal, hydrated conditions cytochrome $f$ is oxidised by light and becomes reduced in darkness. This ceased to happen if the plants had been dried in the sun, which could mean that no electrons were available from the oxidation of water, or possibly that there was a physical uncoupling of cytochrome $f$. Rewetting of the alga immediately resulted in light induced oxidation of cytochrome $f$, indicating that the primary photochemical apparatus had survived the drying process.

The stability of the photosynthetic equipment of Porphyra comes as no surprise to botanists who are familiar with this upper shore alga which daily suffers desiccation yet survives. This work does, however, demonstrate most precisely the nature of this stability. This plant's resilience is even more remarkable when one considers its apparently delicate structure; unlike the robust fucoids, Porphyra forms sheets of tissue just one cell in thickness. For this very reason Porphyra may prove to be an eminently suitable subject for future studies on subcellular responses to extreme environmental conditions.

\section{Correction}

IN the News and Views article "Palindromes in eukaryotic DNA" (Nature, 248, 733; 1974), the sequence in the third paragraph should read:

$$
5^{\prime} \ldots . \mathrm{A} \mathrm{B} \mathrm{C} t \mathrm{C}^{\prime} \mathrm{B}^{\prime} \mathrm{A}^{\prime} \ldots .3^{\prime}
$$

and not as printed. 